\title{
A geometric construction of elliptic conic bundles in $\mathbf{P}^{4}$
}

\author{
by Kristian Ranestad
}

1.9.1997

Dedicated to the memory of Nobuo Sasakura

In the fall of 1996 Abo, Decker and Sasakura [ADS] discovered smooth elliptic conic bundles in $\mathbf{P}^{\mathbf{4}}$. They show that these surfaces all arise as degeneracy loci of four sections of a fixed rank-5 vector bundle on $\mathbf{P}^{\mathbf{4}}$. In particular the family of elliptic conic bundles in $\mathbf{P}^{\mathbf{4}}$ is irreducible.

An elliptic conic bundle in $\mathbf{P}^{\mathbf{4}}$ is a smooth surface $S \subset \mathbf{P}^{\mathbf{4}}$ with a map $S \rightarrow C$ onto an elliptic curve $C$, whose general fibers are embedded as smooth conic sections in $\mathbf{P}^{4}$. In this note I give a construction of the general elliptic conic bundle in $\mathbf{P}^{\mathbf{4}}$ via Cremona transformations. More precisely I construct the general elliptic conic bundle which has a minimal model isomorphic to a smooth quintic elliptic scroll in $\mathbf{P}^{\mathbf{4}}$, i.e. a scroll with minimal selfintersection of a section equal to 1 . I do not prove that every conic bundle have a minimal model like that, but that the general one does.

For any smooth elliptic quintic scroll $S_{5}$ in $\mathbf{P}^{4}$ we find reducible curves $G \cup L$ consisting of a rational normal quartic curve $G$ which meet the scroll in 10 points and a line $L$ which is secant to $G$ and a ruling in the scroll. The quadric hypersurfaces passing through $G \cup L$ define a Cremona transformation of $\mathbf{P}^{4}$, and the image under this transformation of the elliptic scroll is a smooth conic bundle in $\mathbf{P}^{\mathbf{4}}$. Conversely, any elliptic conic bundle in $\mathbf{P}^{\mathbf{4}}$ which have a minimal model isomorphic to a scroll $S_{5}$, contains reducible curves $C_{2} \cup C_{3}$ consisting of a curve $C_{2}$ of degree 6 and genus 4 and a curve $C_{3}$ of degree 9 and genus 6 meeting $C_{2}$ in 6 points three on each of a pair of skew lines. The curve $C_{2}$ lies on a quadric surface, while the curve $C_{3}$ lies on a rational cubic scroll which meet the quadric surface along the two lines. The cubic hypersurfaces through the union of the quadric and the cubic surface defines a Cremona transformation inverse to the one above, and the image of the elliptic conic bundle is a smooth elliptic scroll of degree 5 .

A conic bundle $S$ is not minimal, and the points blown up on a minimal model $S_{5}$ to obtain a surface $S$ may not be chosen in general position. The Cremona transformation gives an extrinsic description in the $\mathbf{P}^{\mathbf{4}}$ of $S_{5}$ of how the points chosen as part of the intersection of $S_{5}$ and a rational normal quartic curve gives rise to a surface $S$. An intrinsic characterization of the 8 points blown up is given in section 3 and compared with the Cremona construction.

\section{Background and minimal models.}

1.1. Let $H$ denote the hyperplane section of $S$, and $K$ the canonical divisor and let $g(H)$ denote the sectional genus, i.e. by adjunction $2 g(H)-2=H^{2}+H \cdot K$.

Recall from [ES, BR]: 
1.2 Proposition. Any elliptic conic bundle $S$ in $\mathbf{P}^{4}$ has degree 8, sectional genus 5 has 8 singular conic fibers. The elliptic threefold scroll $V_{S}$ of planes generated by the conic fibers is a cone, has degree 4 and contains one or two planes which meet every plane in the ruling along a line and the conic bundle in plane quartic curves.

Proof. An elliptic conic bundle is a smooth surface fibered over an elliptic curve in conic sections. By adjunction $|H+K|$ is composed of this fibered structure so $(H+K)^{2}=0$. The Euler characteristic of $S$ is $\chi\left(\mathcal{O}_{S}\right)=0$. The double point formula for surfaces in $\mathbf{P}^{4}$, cf. $[\mathrm{H}]$, yields the relation:

$$
\left(H^{2}\right)^{2}-10 H^{2}-5 H \cdot K-2 K^{2}=0 .
$$

Combined with $(H+K)^{2}=0$ and the adjunction formula, the sectional genus is

$$
g(H)=\frac{1}{2}\left(\left(H^{2}\right)^{2}-7 H^{2}+2\right) .
$$

The Castelnuovo bound for space curves, cf. [H], says

$$
g(H) \leq \frac{1}{4}\left(\left(H^{2}\right)^{2}-1\right)-H^{2}+1,
$$

so $H^{2} \leq 10$. On the other hand eliminating $H \cdot K$ from the double point formula yields $K^{2}=15 H^{2}-2\left(H^{2}\right)^{2}$. Since $K^{2} \leq 0$ we get the lower bound $H^{2} \geq 8$, so $8 \leq H^{2} \leq 10$. For $H^{2}=10$ the sectional genus is 16 , which means that the surface is a complete intersection of a quadric and a quintic hypersurface, while $H^{2}=9$ yields the sectional genus 10, which means that the surface is a complete intersection of two cubic hypersurfaces. Either case is clearly absurd, so $H^{2}=8$ is the only possibility. By adjunction $g(H)=5$, and $K^{2}=-8$ so there are 8 singular conic fibers. The threefold scroll $V_{S}$ of planes generated by the conic sections, have a natural desingularization $V$ in the incidence variety $I$ of points and planes in $\mathbf{P}^{\mathbf{4}} \times \operatorname{Grass}\left(2, \mathbf{P}^{\mathbf{4}}\right)$ :

$$
I=\left\{(x, P) \mid x \in P \subset \mathbf{P}^{\mathbf{4}}, P \in \operatorname{Grass}\left(2, \mathbf{P}^{\mathbf{4}}\right)\right\} \subset \mathbf{P}^{\mathbf{4}} \times \operatorname{Grass}\left(2, \mathbf{P}^{\mathbf{4}}\right)
$$

The preimage of $S$ in $V$ is a smooth surface, in fact isomorphic to $S$, and the projection to the Grassmannian defines the conic bundle structure via this isomorphism. Thus the elliptic base curve $C$ has a natural embedding in $\operatorname{Grass}\left(2, \mathbf{P}^{\mathbf{4}}\right)$. We denote the natural projections, and their restrictions to $S$ by $p: V \rightarrow V_{S}$ and $q: V \rightarrow C$. If $h$ is the pullback on $V$ of the hyperplane in $\mathbf{P}^{4}$ and $f$ is the class of a plane, and $d$ is the degree of $V_{S}$, then $S \cong 2 h+(8-2 d) f$, numerically. The canonical divisor on $V$ is $K_{V} \cong-3 h+d f$, so by adjunction, $\left.K_{S} \cong(-h+(8-d) f)\right|_{S}$. But $K_{S}^{2}=-8$ so $(-h+(8-d) f)^{2} \cdot(2 h+(8-2 d) f)=$ $2 h^{3}+(8-2 d-32+4 d) h^{2} f=4 d-24=-8$, i.e. $d=4$. Clearly the degree of $V_{S}$ is also the Plücker degree of the $C$ in the Grassmannian. So $C$ is an elliptic quartic curve. It spans a $\mathbf{P}^{\mathbf{3}}$. The intersection of this $\mathbf{P}^{\mathbf{3}}$ with the Grassmannian is not proper, in fact it is a quadric surface: The Grassmannian of planes in $\mathbf{P}^{\mathbf{4}}$ is isomorphic to the Grassmannian of lines in $\mathbf{P}^{4}$. Via this isomorphism the curve $C$ correspond to an elliptic quartic surface 
scroll $S_{C}$ in $\mathbf{P}^{\mathbf{4}}$. This scroll spans $\mathbf{P}^{\mathbf{4}}$ only if it is a cone. Via the above isomorphism again $S_{C}$ is a cone if and only if $V_{S}$ is degenerate, while $S_{C}$ is degenerate if and only if $V_{S}$ is a cone. Since $V_{S}$ is not degenerate, it must therefore be a cone. In this case $C$ lies in a Schubert variety corresponding to all planes through a point. This Schubert variety is a quadric 4-fold in a $\mathbf{P}^{\mathbf{5}}$, and may be identified with a Grassmannian of lines in a $\mathbf{P}^{\mathbf{3}}$. The $\mathbf{P}^{\mathbf{3}}$ of $C$ is contained in this $\mathbf{P}^{\mathbf{5}}$ and intersect the quadric hypersurface in a quadric surface. The pencil f $\mathbf{P}^{\mathbf{4}}$ s defining the $\mathbf{P}^{\mathbf{3}}$ of $C$ is generated by two tangent hyperplanes to the quadric 4-fold. These tangent hyperplanes correspond to Schubert varieties of planes through the vertex which meet a given plane through the vertex in a line. If the quadric surface is singular, then the tangent hyperplanes coincide, and all planes in $V_{S}$ meet one plane in a line (through the vertex). If the quadric surface is smooth, then every plane in $V_{S}$ meet two distinct planes in a line. In either case the special planes are contained in $V_{S}$. Since $C$ is the intersection of the quadric surface with another quadric it meets every ruling of either in 2 points. For the rulings belonging to the Grassmannian, this means that the corresponding planes meet along a line. These planes together with one of the special planes, which meet every plane in $V_{S}$ in line, span a hyperplane. Therefore the intersection of the special plane with $S$ is a curve of degree at most 4 . It is clearly a bisection, i.e. it meets every ruling in two points. On the other hand through every line through the vertex there are two planes of the ruling, since $C$ is of type $(2,2)$. Therefore the special plane meet $S$ in a curve of degree $4 . \square$

1.3 Minimal models. The singular conic fibers of the map $q: S \rightarrow C$, which defines the conic bundle structure, consists of pairs of $(-1)$-lines on the surface. Since there are 8 singular fibers, there are therefore $2^{8}$ different minimal models for $S$, and each minimal model is a $\mathbf{P}^{\mathbf{1}}$-bundle over an elliptic curve. On a $\mathbf{P}^{\mathbf{1}}$-bundle the parity of the selfintersection of all sections coincide. The choice of (-1)-line to blow down in a singular conic on $S$ corresponds precisely to this choice of parity downstairs. There are therefore $2^{7}$ minimal models of each parity, which we call even or odd accordingly. Any section of the map $q$ will together with 8 exceptional curves and the pullback of the Picard group of the elliptic base curve $C$ generate the Picard group of $S$. Given a section $C_{0}$ with selfintersection $C_{0}^{2}=a$ on $S$, one may always blow down all the $(-1)$-lines on $S$ which do not meet $C_{0}$ to get a minimal model. Thus the selfintersection of $C_{0}$ on the minimal model is unchanged. Numerically $H \cong 2 C_{0}+(4-a) F-E_{1}-\ldots-E_{8}$, so $H \cdot C_{0}=2 a+4-a=$ $a+4=C_{0}^{2}+4$. Since $C_{0}$ is elliptic the degree is at least 3 so the selfintersection is at least -1. In fact every section $C_{0}$ with selfintersection $C_{0}^{2}=-1$ is a plane cubic curve on $S$. On the other hand any plane cubic curve on $S$ is contained in a pencil of hyperplanes, so it is at most a bisection on $S$. If it was a bisection, the residual moving part of degree 5 of the pencil of hyperplane sections is supported on a finite number of fibers. But this is impossible since the residual moving part would have to include an exceptional line and these do not move. Therefore every plane cubic curve on $S$ is a section with selfintersection -1 . Thus

1.4 Lemma. $S$ has $2^{7}$ odd minimal models, and the minimal selfintersection of a section is in each case 1 or -1 . The sections with selfintersection -1 correspond precisely to the plane cubic curves on $S$. 
1.5 Remark. With minimal selfintersection of a section equal to 1 , the minimal model is isomorphic to a smooth elliptic quintic scroll in $\mathbf{P}^{\mathbf{4}}$. Since the family of elliptic conic bundles is irreducible, and conic bundles with plane cubic curves cannot degenerate to conic bundles without plane cubic curves, it is clear that the conic bundles with a minimal model isomorphic to a smooth quintic scroll are the general ones on the family. On the other hand it is not clear whether every conic bundle $S$ has a minimal model isomorphic to a smooth quintic scroll.

\section{The Cremona construction}

2.1. Consider an elliptic quintic scroll $S_{5}$ and a general complete intersection of two cubics containing it. The residual to $S_{5}$ is a smooth Veronese surface $V_{4}$, the smooth projection of a Veronese surface in $\mathbf{P}^{5}$. The curve $D=S_{5} \cap V_{4}$ is linearly equivalent to $H-K$ on each of the two surfaces. Therefore it is a plane quintic curve on the plane (i.e. of degree 10 on the Veronese surface), while it is a 3 -section on the scroll. Now, pick a general member of the ruling of $S_{5}$. It is a line $L$, which meet $D$ in 3 distinct points. Pick 2 of the three points, and consider the rational quartic curves on $V_{4}$ which pass through these two points. These curves form a web, while the hyperplane sections of $V_{4}$ through the two points form a net, so the general member of the web spans $\mathbf{P}^{4}$. Pick a smooth member $G$ which spans $\mathbf{P}^{4}$. The union $B=L \cup G$ has arithmetic genus 1 , and has ideal generated by the 5 quadric Pfaffians of a skewsymmetric $5 \times 5$ matrix with linear entries. $B$ is a degeneration of an elliptic normal quintic curve. The 5 quadrics which cut out $B$ define a Cremona transformation $\phi_{B}$ of $\mathbf{P}^{4}$, cf. [S]. This Cremona transformation collapses the secant lines to $B$. More precisely, let $U \rightarrow \mathbf{P}^{\mathbf{4}}$ be the blowup of $\mathbf{P}^{\mathbf{4}}$ in the ideal of $B$, and let $Z_{U}$ be a scheme of length 2 on $U$ whose image $Z$ in $\mathbf{P}^{4}$ also has length 2 . Then $Z_{U}$ is mapped to a point by $\phi_{B}: U \rightarrow \mathbf{P}^{4}$, if and only if the line $L_{Z}$ generated by $Z$ intersect $B$ in a scheme of length 2 . From various projections of $B$ to a plane one easily derives that the secants of $B$ form a reducible hypersurface. The secants to $G$ is a cubic hypersurface, while the lines joining $L$ and $G$ form a quadric hypersurface, singular along $L$.

2.2. We restrict $\phi_{B}$ to $S_{5}$. Since $G$ meets $D$ in 10 points, and 2 of them lie on $L$, we see that $B$ intersect $S_{5}$ in $L$ and in 8 additional points. Therefore $\phi_{B}$ restrict to $S_{5}$ as a map defined by a linear system $|2 H-L|$ with assigned basepoint at these 8 points. Let $S$ denote the blow up of $S_{5}$ in these 8 points, and let $E, \ldots, E_{8}$ be the exceptional curves. The linear system on $S$ becomes

$$
|h|=\left|2 H-L-E_{1}-\ldots-E_{8}\right|,
$$

where $H$ and $L$ are the pullback of the corresponding divisors on $S_{5}$. For very ampleness of $|h|$, we investigate the intersection of secants to $B$ with $S_{5}$. Consider first a subscheme $Z$ of length 2 on $S_{5}$ which does not intersect $B$ and for which the line $L_{Z}$ generated by $Z$ is a secant line to $B$. Since $L_{Z}$ has a scheme of length 4 of intersection with $S_{5} \cup V_{4}$, and this union is a complete intersection of two cubics, it must be contained in the union of the two surfaces. But $V_{4}$ contains no lines, so $L_{Z}$ must be a ruling of $S_{5}$. Thus $Z$ is collapsed by $\phi_{B}$ only if $L_{Z}$ is a ruling of $S_{5}$, which is a secant line to $G$.

Next, consider a subscheme $Z_{U}$ of length 2 on $S$ whose image $Z$ on $S_{5}$ has length 2 and intersect $B$ in a point. If $Z_{U}$ is collapsed to a point, the line $L_{Z}$ generated by $Z$ must be 
a secant to $B$. There are two possibilities. If $Z$ does not intersect $L$, then $L_{Z}$ must be a trisecant to $S_{5}$, tangent to $S_{5}$ at a point of intersection with $G$. In addition it must be a secant line to $G$, so it intersects the union $S_{5} \cup V_{4}$ in a scheme of length at least 4 . As above it must be a member of the ruling of $S_{5}$, which is at the same time a secant to $G$. If $Z$ intersect $L$, the line $L_{Z}$ must again be a trisecant to $S_{5}$, tangent to $S_{5}$ at a point on $L$. It is also a secant to $B$ so it must intersect $G$ at some point disjoint from $L$. Again $L_{Z}$ is a 4 -secant to the union of the two surfaces, so it must be a member of the ruling, i.e. it must coincide with $L$, absurd. Finally we consider a subscheme $Z_{U}$ of length 2 of $S$ whose image $Z$ on $S_{5}$ is contained in $B$. If $Z$ has length 2 then $L_{Z}$ is a 4 -secant to $S_{5}$ tangent at two points, but this is impossible, since $S_{5}$ is cut out by cubics, unless $L_{Z}$ is a member of the ruling. If $Z$ is a point, it is one of the 8 points of intersection of $G$ and $S_{5}$ outside $L$. But, the Cremona transformation has no basepoints on the blowup, so every exceptional curve of $S$ is embedded as a line, and has therefore no double points.

As a conclusion

2.3 Proposition. The Cremona transformation defines an embedding of $S$ as soon as $G$ meets the scroll $S_{5}$ in 10 distinct points, and no secant of $G$ except $L$ coincide with a ruling of $S_{5}$.

2.4 The converse Cremona transformation. The secants of $B$ have two components, the secant variety of $G$, a cubic hypersurface $V_{3}$, and the join of $L$ and $G$ which is a rank 3 quadric $V_{2}$, singular along $L$. Both of these are collapsed to surfaces by the Cremona transformation. The map defined by all quadrics through $G$ maps $V_{3}$ to a Veronese surface in $\mathbf{P}^{\mathbf{5}}$, the 5 quadrics of the Cremona transformation projects this Veronese surface from a point on the surface, the image of the secant line $L$, to a cubic scroll $S_{3}$ in $\mathbf{P}^{4}$. The quadric $V_{2}$ is one of the quadrics which define the Cremona transformation, so its image is contained in a hyperplane. The pencil of lines joining $L$ and a fixed point on $G$ sweep out a plane. This plane and $G$ lie in 3 quadrics so the image of the pencil is a line. Clearly distinct points on $G$ generate disjoint pencils with disjoint image lines by the Cremona, so the image of $V_{2}$ is a smooth scroll, i.e. a quadric surface $S_{2}$. The opposite ruling on $S$ is formed by the pencils of lines joining $G$ and a fixed point on $L$. The intersection of the surfaces $S_{2}$ and $S_{3}$ is formed by the two pencils of secants to $G$ from the two points of intersection between $G$ and $L$. Thus $S_{2} \cap S_{3}$ form two skew lines. The cubic scroll $S_{3}$ is smooth and the directrix lie in the hyperplane of $S_{2}$ and intersect $S_{2}$ properly in two points.

The converse Cremona transformation is defined by the cubic hypersurfaces through the quadric surface $S_{2}$ and the cubic scroll $S_{3}$. The union $T=S_{2} \cup S_{3}$ is a degenerate quintic elliptic scroll, its ideal is generated by 5 cubics. The converse Cremona collapses subschemes of length 2 precisely when the line generated by the subscheme intersect $T$ in a scheme of length 3, i.e. is a trisecant to $T$. In the case of a smooth elliptic quintic scroll, the trisecants are trisecants of an elliptic pencil of plane cubic curves on the surface. In our degenerate case, the plane cubic curves belong to two families: the pencil of planes through the directrix of $S_{3}$ inside the span of $S_{2}$, this family of planes form a line in the Grassmannian of planes and is mapped to the line $L$ by the Cremona transformation. The other family of plane cubic curves on $T$, are defined by the by planes through the rulings 
of $S_{2}$ which are secants to $S_{3}$. Each secant line on $S_{3}$ is a secant to a unique conic section on $S_{3}$. This conic section spans a plane which therefore intersect $T$ in a cubic curve. The family of planes defined this way form a rational curve in the Grassmannian of planes, and it has two planes in common with the linear pencil of planes. Each of these planes are collapsed to a point by the Cremona transformation, and the family is mapped to the rational normal quartic curve $G$.

2.5. The surface $S$ intersect $S_{2}$ and $S_{3}$ in curves $C_{2}$ and $C_{3} . C_{2}$ is part of a hyperplane section, it is the section defined $V_{2}$, since this quadric is singular along $L, C_{2}$ is equivalent to $h-L$, i.e. a curve of degree 6 and genus 4 . Since a curve of degree 6 and genus 4 is a complete intersection $(2,3)$ in a $\mathbf{P}^{3}$, we see that $S_{2}$ is determined by $C_{2}$. The curve $C_{3}$ is defined by the cubic $V_{3}$, since this hypersurface is the secant variety of $G$, it contains $L$ and is singular in all the eight points which are blown up. Thus

$$
C_{3} \equiv 3 H-L-2 E_{1}-\ldots-2 E_{8} \equiv 3 h-H-C_{2} \equiv 2 h-H+L .
$$

We see that on $S$, the curve $C_{3}$ is residual to $H-L$ in a quadric. But $H-L$ moves in a net and the general member is a smooth elliptic curve of degree 7 on $S$. An elliptic curve of degree 7 lies on at least one quadric, so $C_{3}$ is the baselocus on $S$ of the quadrics containing the curves in the net $|H-L|$. Thus the quadrics form a net and define a cubic scroll in $\mathbf{P}^{4}$. This scroll of course coincides with $S_{3}$. $C_{3}$ has degree 9 and genus 6 , so it must be of type $5 l-e$ on the scroll, when we think of the scroll as the blowup of the plane in a point, $l$ is the class of a line and $e$ the class of the exceptional curve, the directrix on the scroll. Since $C_{2} \cdot C_{3}=6$ the curve $C_{3}$ intersect $C_{2}$ in 3 points on each of the common rulings of the scrolls $S_{2}$ and $S_{3}$.

2.6. We may recover this converse Cremona directly from $S$ for the general conic bundle $S$ with minimal model $S_{5}$ isomorphic to a smooth elliptic quintic scroll. For this we start by considering sections $C_{0}$ of $S_{5}$ with selfintersection 3. By 1.4 such curves always exist and moves in a net without basepoints on $S_{5}$. We denote the strict transform of $C_{0}$ on $S$ also by $C_{0}$. It has degree 7 in the embedding in $\mathbf{P}^{4}$ and the general member of the net $\left|C_{0}\right|$ on $S$ is smooth. Each curve in $\left|C_{0}\right|$ lie on at least one quadric. Since $S$ does not lie in any quadric hypersurface, any curve $C_{3}$ in $\left|2 h-C_{0}\right|$ must lie in a net of quadrics. $C_{3}$ has degree 9 , so the net must define a cubic scroll: Since the general curve $C_{0}$ is smooth, the general quadric which contain $C_{3}$ is irreducible. Therefore the net of quadrics define at most a surface. Since the intersection of the quadrics contains a curve of degree 9 the intersection is at least a surface, so the intersection must be a surface. It lies on three quadrics so it is a possibly degenerate cubic scroll $S_{3}$.

For the general surface $S$ and linear system $\left|C_{0}\right|$ we may assume that the cubic scroll is smooth, in fact that is the case in the construction above. In the smooth scroll, $C_{3}$ must be equivalent to $5 l-e$, since it has degree 9 and arithmetic genus 6 . So it contains the directrix as a component or meets it in a unique point $x$. By genericity we assume that $C_{3}$ meets the directrix properly in $x$. Since $\left|C_{0}\right|$ is a net without basepoints, the intersection of $S$ with the cubic scroll is precisely $C_{3}$. Therefore the directrix and $L$ intersect only in $x$, and they span a unique hyperplane. Residual to $L$ in this hyperplane is a curve $C_{2}$ on $S$ which has degree 6 and arithmetic genus 4 . This is a complete intersection $(2,3)$. By 
genericity again we may assume that the quadric surface $S_{2}$ containing $C_{2}$ is smooth, in fact that is the case in the above example. The hyperplane of $S_{2}$ intersect the cubic scroll $S_{3}$ along the directrix and two rulings. Since the directrix does not lie on the quadric surface, and the intersection $C_{2} \cdot C_{3}=6$ the two rulings of the cubic scroll must also be rulings of the quadric surface $S_{2}$. The union $S_{3} \cup S_{2}$ form a degenerate elliptic quintic scroll. The cubics through $S_{3} \cup S_{2}$ define a Cremona transformation of $\mathbf{P}^{4}$, cf. [S]. This transformation maps $S$ onto a smooth quintic elliptic scroll: In fact the restriction of the transformation to the surface $S$ is a linear system

$$
|H|=\left|3 h-C_{2}-C_{3}\right|=\left|3 h-(h-L)-\left(2 h-C_{0}\right)\right|=\left|C_{0}+L\right|
$$

of sections with selfintersection 5 coming from a minimal model isomorphic to a smooth quintic elliptic scroll. Such a linear system is very ample on the minimal model obtained by blowing down all exceptional lines that does not intersect the general curve in the linear system.

We may conclude:

2.7 Proposition. If $S$ is a general elliptic conic bundle with minimal model isomorphic to a smooth quintic elliptic scroll, then $S$ is obtained by a Cremona transformation from such a scroll.

2.8 Remark. The construction of elliptic conic bundles in $\mathbf{P}^{\mathbf{4}}$ described in [ADS] shows in particular that the family of all these conic bundles is irreducible. In the construction of conic bundles from elliptic quintic scrolls in 2.2 , it is clear that the genericity conditions of 2.6 are satisfied. Since these conditions are open it follows from 2.7 and remark 1.5 that the general elliptic conic bundle appear via a Cremona transformation.

\section{Construction via linear systems and plane quartic curves}

3.1. Again we restrict the attention to conic bundles $S$ with minimal model isomorphic to a smooth quintic scroll $S_{5}$. In this case as we saw in 2.2 that the linear system of hyperplane sections on $S$ can be written as

$$
|h|=\left|2 H-L-E_{1}-\ldots-E_{8}\right|,
$$

where $H$ is the pullback of a hyperplane section on $S_{5}$, the divisor $L$ is the pullback of a ruling and the $E_{i}$ are exceptional curves. This linear system is special, in fact the 8 points blown up to obtain $S$ from $S_{5}$ may not be chosen generally. By Riemann Roch one computes that $h^{0}\left(\mathcal{O}_{S}(h)\right)=5$ precisely when $h^{1}\left(\mathcal{O}_{S}(h)\right)=1$. The open condition of very ampleness for $|h|$ requires that among the 8 points on $S_{5}$ no 2 of the points are infinitely close or lie on the same ruling of $S_{5}$. The more important closed condition that is required for $h^{0}\left(\mathcal{O}_{S}(h)\right)=5$ can be understood by looking at a plane quartic curve on $S$. Recall the projection map $q: S \rightarrow C$ which defines the conic bundle structure on $S$. The blowup map $\pi: S \rightarrow S_{5}$ is a factor of the projection $q$, therefore the curve $C$ now have a natural embedding in the Grassmannian of lines in the $\mathbf{P}^{4}$ of $S_{5}$. We denote Plücker divisor on $C$ of this embedding by $\gamma$.

Now, on the conic bundle $S$ we know from 1.2 that there is a plane quartic curve linearly equivalent to $A \equiv h-q^{*} \alpha$ for some divisor $\alpha$ of degree 2 on $C$. Since $D$ is a plane quartic 
curve in the embedding by $h$ this linear system restricts as the canonical linear series on $S$. The canonical series on $S$ is $K \equiv-2 H+q^{*} \gamma+E_{1}+\ldots+E_{8}$. The adjoint series to $A$ is $q^{*}(\gamma-\alpha)-L \equiv q^{*} \beta$ the pullback of a divisor of degree 2 on $C$, and the restriction of $h$ to $A$ coincides with this only if $h-q^{*} \beta$ restricts trivially to $A$. When $q^{*} \alpha$ and $q^{*} \beta$ are distinct then $h^{1}\left(\mathcal{O}_{S}\left(h-q^{*} \beta-A\right)\right)=0$, so $h-q^{*} \beta$ restricts trivially to $A$ if and only if there is a curve $A_{1} \equiv h-q^{*} \beta$ on $S$. On $S_{5}$ this means that the 8 points blown up are precisely the complete intersection $\pi(A) \cap \pi\left(A_{1}\right)$ on $S_{5}$. The argument now applies also to $A_{1}$, so when $\alpha$ and $\beta$ are not equivalent as divisors on $C$, then $S$ have the two plane quartic curves $A$ and $A_{1}$ and these determine the exceptional curves completely. If $\alpha \equiv \beta$ then $A$ and $A_{1}$ may coincide. In fact in this case the 8 points on $\pi(A)$ belong to the linear series $|\pi(A)|_{\pi(A)}$, which are not the intersection of a curve $\pi\left(A_{1}\right)$ linearly equivalent to $\pi(A)$. This is possible: Since $S_{5}$ is irregular, the restriction of $|\pi(A)|$ to $\pi(A)$ is not complete.

3.2 This intrinsic description of the linear system $|h|$ on $S$, may also be related to the Cremona construction in section 2 .

In the Cremona construction a plane quartic curve $A$ on $S$ is defined via the transformation by a pencil of quadrics which contain $B$ and 2 additional rulings of $S_{5}$. Following the construction of the linear system $|h|$ we start with a ruling $L$ and a smooth curve $A_{0} \equiv$ $2 H-L-q^{*} \alpha$ on $S_{5}$ for some divisor $\alpha$ of degree 2 on the elliptic base curve. The union $A_{0} \cup L$ has degree 8 and arithmetic genus 4 and lies in a pencil of quadrics. By proving that the three quadrics through $A_{0}$ define a complete intersection, one can show that the pencil through $A_{0} \cup L$ defines a smooth complete intersection. Furthermore, $A_{0} \cup L$ lies on many cubic hypersurfaces which does not contain $S_{5}$. A general cubic through $A_{0} \cup L$ define together with the pencil of quadrics a complete intersection $(2,2,3)$. In this complete intersection $A_{0} \cup L$ is linked to a smooth rational normal quartic curve $G$. In fact any curve in $|G|$ on the complete intersection of the quadrics, are linked to to $A_{0} \cup L$ in a complete intersection $(2,2,3)$. The line $L$ is a secant to $G$, so the quadrics through $G \cup L$ define a Cremona transformation as above. $G$ intersect $A_{0}$ in 8 points, which are blown up on $S_{5}$ by the Cremona transformation.

Consider the set of cubics through $S_{5}$ and $G$. There are precisely a pencil containing both. This pencil defines a complete intersection containing $S_{5}$ and a quartic surface $S_{4}$. The quartic surface $S_{4}$ is a Veronese surface or a degeneration of a Veronese surface. Any such degeneration is reducible and does not contain rational normal quartic curves, so since $G$ is smooth $S_{4}$ must be a smooth Veronese surface. Since there is precisely a pencil of cubics through $S_{5} \cup G$, the Veronese surface $S_{4}$ is completely determined by $G$. By construction precisely a pencil of the quadrics through $G \cup L$ contain $A_{0}$, while the Cremona transformation has degree 4 on $A_{0}$, so $A_{0}$ is canonically embedded as a plane quartic curve by the Cremona transformation. Therefore the above argument applies to give the intrinsic description of the 8 points blown up on $S_{5}$. In particular it follows that $A_{0}$ may or may not be the only plane quartic curve on $S$ depending on the choice of $L$ and the divisor $\alpha$.

3.4 Open problems. The constructions in section 2 and 3 give the general elliptic conic bundle in $\mathbf{P}^{\mathbf{4}}$. They leave open whether every conic bundle with minimal model an elliptic quintic scroll can be constructed this way. In particular, are there conic bundles with only 
one plane quartic curve? The argument also leaves open the problem whether there are conic bundles $S$ which do not admit $S_{5}$ as a minimal model, i.e. for which every minimal model have a -1 -section.

\section{References}

[ADS] Abo, H., Decker W., Sasakura N.: An elliptic conic bundle in $\mathbf{P}^{4}$ arising from a stable rank-3 vector bundle. alg-geom/9708023 (1997)

[BR] Braun R.,Ranestad, K.: Conic bundles in projective fourspace to appear in Proceedings of the Europroj annual conference 1994, Marcel Dekker

[ES] Ellia, P., Sacchiero, G.: Smooth surfaces of $\mathbf{P}^{\mathbf{4}}$ ruled in conics. to appear in Proceedings of the Europroj annual conference 1993, Marcel Dekker

[H] Hartshorne, R.: Algebraic Geometry. Berlin, Heidelberg, New York: Springer (1977).

[S] Semple, J. G.: Cremona transformations of space of four dimensions by means of quadrics and the reverse transformations, Phil. Trans. Royal Soc. London, Series A, 228 (1929), 331-376.

Authors address:

Matematisk Institutt, P.b. 1053 Blindern, N-0316 Oslo 3, Norway

email: ranestad@math.uio.no 